\title{
SPECTRAL MAPPING THEOREMS FOR WEYL SPECTRUM AND ISOLATED SPECTRAL POINTS
}

\author{
JIANGTAO YUAN AND CAIHONG WANG
}

Abstract. Spectral mapping theorems for Weyl spectrum and isolated spectral points were discussed by Gramsch, Lay and Oberai, etc. In this paper, $\mathscr{L}(\mathscr{X})$ means the space of all bounded linear operator on an infinite-dimensional complex Banach space $\mathscr{X}, f \in \mathscr{H}(\sigma(T))$ means $f$ is holomorphic on an open set $\mathscr{U}$ containing the spectrum $\sigma(T)$, and $f \in \mathscr{H}_{\text {lnc }}(\sigma(T))$ means $f$ is holomorphic and locally nonconstant. Firstly, it is shown that, if $T \in \mathscr{L}(\mathscr{X})$ and $f \in \mathscr{H}(\sigma(T))$, then $(1) \sigma_{u w}(f(T)) \subseteq f\left(\sigma_{u w}(T)\right)$ where $\sigma_{u w}(T)$ means the upper semi-Weyl spectrum; (2) $\sigma_{u w}(f(T)) \supseteq f\left(\sigma_{u w}(T)\right)$ is equivalent to the assertion that $T$ is of stable sign index on $\rho_{u f}(T)$ where $\rho_{u f}(T)$ means the upper semi-Fredholm resolvent. Secondly, let $T \in \mathscr{L}(\mathscr{X})$, (1) if $f \in \mathscr{H}_{l n c}(\sigma(T))$ or $T$ is polaroid, then $\sigma(f(T)) \backslash \pi_{00}(f(T)) \subseteq f\left(\sigma(T) \backslash \pi_{00}(T)\right)$; (2) if $T$ is isoloid, then $\sigma(f(T)) \backslash \pi_{00}(f(T)) \supseteq f\left(\sigma(T) \backslash \pi_{00}(T)\right)$. Some two-out-of-three results on spectral mapping theorems and Weyl type theorems are also given. At the end, an example is provided which implies that the conditions " $f \in \mathscr{H}_{\text {Inc }}(\sigma(T))$ ", " $T$ is polaroid" and " $T$ is isoloid" are crucial and inevitable.

Mathematics subject classification (2010): 47A53, 47A10.

Keywords and phrases: Weyl spectrum, B-Weyl spectrum, spectral mapping theorem, isolated spectral point, Weyl theorem.

\section{REFERENCES}

[1] P. AiEnA, Fredholm and local spectral theory, with application to multipliers, Kluwer Acad. Publishers 2004.

[2] P. AienA, Classes of Operators Satisfying a-Weyl's theorem, Studia Math. 169 (2005), 105-122.

[3] P. AIEnA, Quasi-Fredholm operators and localized SVEP, Acta Sci. Math. (Szeged) 73 (2007), 251263.

[4] P. Aiena, E. Aponte And E. Balzan, Weyl type theorems for left and right polaroid operators, Integral Equations Operator Theory 66 (2010), 1-20.

[5] P. Aiena, J. R. Guillen And P. PenA, A unifying approach to Weyl type theorems for Banach space operators, Integral Equations Operator Theory 77 (2013), 371-384.

[6] M. BERKANI, On a class of quasi-Fredholm operators, Integral Equations Operator Theory 34 (1999), 244-249.

[7] M. BERKANI, Restriction of an operator to the range of its powers, Studia Math. 140 (2000), 163-175.

[8] M. BERKANI, Index of B-Fredholm operators and generalization of a Weyl theorem, Proc. Amer. Math. Soc. 130 (2002), 1717-1723.

[9] M. Berkani AND A. Arroud, Generalized Weyl's theorem and hyponormal operators, J. Aust. Math. Soc. 76 (2004), 291-302.

[10] M. Berkani, M. SARIH, On semi B-Fredholm operators, Glasgow Math. J. 43 (2001), 457-465.

[11] R. E. CURTO AND Y. M. HAN, Generalized Browder's and Weyl's theorems for Banach space operators, J. Math. anal. Appl. 336 (2007), 1424-1442.

[12] B. P. Duggal, Polaroid operators satisfying Weyl's theorem, Linear Algebra Appl. 414 (2006), no. $1,271-277$.

[13] B. Gramsch And D. C. Lay, Spectral mapping theorems for essential spectra, Math. Ann. 192 (1971), 17-32. 
[14] W. Y. LEE AND S. H. LEE, A spectral mapping theorem for the Weyl spectrum, Glasgow Math. J. 38 (1996), 61-64.

[15] K. K. OberaI, On the Weyl spectrum II, Illinois J. Math. 21 (1977), 84-90.

[16] C. Schmoeger, On operators T such that Weyl's theorem holds for $f(T)$, Extracta Math. 13 (1998), $27-33$.

[17] J. T. YUAn AND G. X. Ji, On (n,k)-quasiparanormal operators, Studia Math. 209 (2012), 289-301.

[18] H. Zguitti, A note on generalized Weyl's theorem, J. Math. Anal. Appl. 316 (2006), 373-381. 